\title{
COMMENTARY
}

\section{A new hope for patients suffering from multiple myeloma}

Jinhua Yu and Yao Li

See related research by Atsuta et al., http://stemcellres.com/content/4/5/111

\begin{abstract}
Multiple myeloma is a common hematological malignancy that leads to the occurrence of bone lesions. The combination of traditional cytotoxic and novel agents is usually taken to treat multiple myeloma-related bone diseases. However, the curative effect is not very satisfactory. Stem cell-based therapy has been recently introduced and investigated, which represents a new frontier in the treatment of multiple myeloma. In a recent interesting study, Dr Atsuta and colleagues provide a new insight into the effects of mesenchymal stem cells on multiple myeloma via Fas/ Fas ligand pathway, which rekindles the fire of hope for those patients suffering from multiple myeloma.
\end{abstract}

Over the last decades, multiple myeloma (MM) remains an incurable hematological malignancy and novel therapeutic approaches are urgently required. Bone disease is one of the most frequent problems in MM patients, and the balance between formation (osteoblastogenesis) and resorption (osteoclastogenesis) in bone is absent in MM patients. In this regard, traditional cytotoxic and novel agents that can inhibit osteoclast activity and/or activate osteoblasts are used to treat MM-related bone diseases. However, the curative effect is still poor and patients with MM will develop refractory diseases. Therefore, it is necessary to explore new methods to cure MMrelated bone diseases. Recently, the use of mesenchymal stem cells (MSCs) to treat MM-related bone diseases has received considerable attention, which represents a new approach to the treatment of multiple myeloma [1]. The present article from Dr Atsuta and colleagues has revealed that MSCs can act on multiple myeloma cells via the Fas/Fas ligand (FasL) pathway [1].

\footnotetext{
* Correspondence: yuziyi_yjh@hotmail.com

Institute of Stomatology, Nanjing Medical University, 140 Hanzhong Road, Nanjing, Jiangsu 210029, China
}

The Fas/FasL pathway plays an important role in inducing cell apoptosis [2], but it is unclear whether Fas/FasL is related to the influence of MSCs on MM cells. In this paper, the authors provide new insights into the effects of MSCs on MM cells in vitro and in vivo via altering Fas/FasL expression. In the MM mouse model, treatment of MSCs with highly expressed FasL (FasL high MSCs) shows remarkable inhibitory effects on MM growth, bone resorption in the lumbus, and tumor metastasis in the lungs and kidneys [1]. The authors' findings thus seem to open up a promising strategy for upcoming anti-MM treatments.

The use of MSCs in treating cancer is still controversial, however, with some studies finding that stem cells force tumors to enter programmed cell death, while others reveal that stem cells actually promote tumor growth including MM by inducing infiltration of new blood vessels $[3,4]$. Therefore, when considering the potential clinical utilization of MSC-based therapy, an unavoidable question is: why do FasL high MSCs suppress the proliferation of MM? The authors have indicated that MSC/FasL-induced MM apoptosis may explain MSC-based inhibition of MM growth. Thus, the open question at the basis of this study is: what are the differences between naïve MSCs and FasL high MSCs? No matter what these differences are, one fact is clear from the reported findings - MSCs from different sources or microenvironments can differentially influence the progression of MM, and the surrounding environment can determine the behavior of MSCs.

Although MSC-based cytotherapy has established a novel concept for the treatment of MM-related bone diseases [5], whether systemic and intrabone cytotherapeutic strategies are effective needs to be explored extensively. $\mathrm{Li}$ and colleagues have shown that systemic injections of MSCs restrain MM disease progression, while intrabone injections of MSCs not only inhibit tumor growth in the bone but also effectively promote bone formation during disease/remission and delay MM 
relapse [6]. However, the mechanisms of action by which MSC cytotherapy stimulates bone formation and inhibits MM-induced bone tumor growth are only partially understood. In the present paper, FasL high MSCs demonstrate significant inhibition, while FasL null MSCs enhance MM growth, suggesting that the levels of FasL expression in MSCs determine, at least in part, the effects of MSCs on cancer growth. Another important question is: can these FasL high MSCs be used to treat MM patients? This question may be the biggest one facing the cytotherapy of MM in the 21st century: what is it really like inside the tumor?

In conclusion, Dr Atsuta and colleagues have provided a new way of thinking about the potential utilization of MSCs against MM cells, which represents a significant contribution to the treatment of MM bone diseases in the future.

\section{Abbreviations}

FasL: Fas ligand; FasL high MSC: Mesenchymal stem cells with highly

expressed Fas ligand; MM: Multiple myeloma; MSC: Mesenchymal stem cell.

\section{Competing interests}

The authors declare that they have no competing interests.

\section{Published: 27 November 2013}

\section{References}

1. Atsuta I, Liu S, Miura Y, Akiyama K, Chen C, An Y, Shi S, Chen FM: Mesenchymal stem cells inhibit multiple myeloma cells via the Fas/Fas ligand pathway. Stem Cell Res Ther 2013, 4:111.

2. Mazar J, Thomas M, Bezrukov L, Chanturia A, Pekkurnaz G, Yin S, Kuznetsov SA, Robey PG, Zimmerberg J: Cytotoxicity mediated by the Fas ligand (FasL)-activated apoptotic pathway in stem cells. J Biol Chem 2009, 284:22022-22028.

3. Roccaro AM, Sacco A, Maiso P, Azab AK, Tai YT, Reagan M, Azab F, Flores LM, Campigotto F, Weller E, Anderson KC, Scadden DT, Ghobrial IM: BM mesenchymal stromal cell-derived exosomes facilitate multiple myeloma progression. J Clin Invest 2013, 123:1542-1555.

4. Ciavarella S, Caselli A, Savonarola A, Tamma AV, Tucci M, Silvestris F: Cytotherapies in multiple myeloma: a complementary approach to current treatments? Expert Opin Biol Ther 2013, 13:S23-S34.

5. Fu J, Wang P, Zhang X, Ju S, Li J, Li B, Yu S, Zhang J: Myeloma cells inhibit osteogenic differentiation of mesenchymal stem cells and kill osteoblasts via TRAIL-induced apoptosis. Arch Med Sci 2010, 6:496-504

6. Li X, Ling W, Khan S, Yaccoby S: Therapeutic effects of intrabone and systemic mesenchymal stem cell cytotherapy on myeloma bone disease and tumor growth. J Bone Miner Res 2012, 27:1635-1648. 